\title{
Increasing Crew Autonomy for Long Duration Exploration Missions: Self-Scheduling
}

\author{
Jessica J. Marquez \\ Steven Hillenius \\ NASA Ames Research Center \\ M/S 262-2 \\ Moffett Field, CA 94035 \\ jessica.j.marquez@nasa.gov \\ (650) 604-6364 \\ steven.r.hillenius@nasa.gov \\ (650) 604-2888
}

\author{
Bob Kanefsky \\ Jimin Zheng \\ San Jose State Research \\ Foundation \\ M/S 262-4 \\ Moffett Field, CA 94035 \\ bob.kanefsky@nasa.gov \\ (650) 604-3514 \\ jimin.zheng@nasa.gov \\ (650) 604-5831
}

\author{
Ivonne Deliz \\ ASRC Federal \\ $M / S$ 262-4 \\ Moffett Field, CA 94035 \\ ivy.deliz@nasa.gov \\ (650) 604-5616 \\ Marcum Reagan \\ NASA Johnson Space Center \\ 2101 NASA Parkway, XM \\ Houston, TX 77058 \\ marcum.l.reagan@nasa.gov
}

(281) 244-7489

\begin{abstract}
Over the last three years, we have been investigating the operational concept of crew self-scheduling as a method of increasing crew autonomy for future exploration missions. Through Playbook, a planning and scheduling software tool, we have incrementally enabled the capability for Earth analog mission crews to modify their schedules at various levels of complexity. Playbook allows the crew to create new activities from scratch, add activities or groups of activities from a Task List, and reschedule or reassign flexible activities. The crew is also able to identify if plan modifications create violations, i.e., plan constraints not being met. This paper summarizes our observations with qualitative evidence from four NASA Extreme Environment Mission Operations (NEEMO) analog missions that supported self-scheduling as a feasible operational concept.
\end{abstract}

\section{TABLE OF CONTENTS}

1. INTRODUCTION 1

2. CRew AUtonomy................................................... 1

3. ENABLING SELF-SCHEDULING ........................... 2

4. INITIAL ASSESSMENT: NEEMO .......................... 5

5. LESSONS LEARNED .......................................... 6

6. FUTURE EVALUATIONS ........................................ 8

7. CONCLUDING REMARKS ..................................... 8

ACKNOWLEDGEMENTS .......................................... 9

REFERENCES..................................................... 9

BIOGRAPHY ........................................................... 9

\section{INTRODUCTION}

As future exploration missions seek to send humans farther from Earth for longer durations, NASA must also further its understanding of how to enable astronauts conducting work during these types of missions. In these scenarios, crew will be required to operate more autonomously, since communication with Earth will be both delayed (from seconds to minutes) and intermittent. Crew will only have limited opportunities to ask ground teams for clarifications and guidance on assigned tasks. Furthermore, crew may also have unique, in-situ knowledge about how to execute tasks, U.S. Government work not protected by U.S. copyright. in which they may not be able to communicate to ground in a timely manner. One key factor in allowing astronauts to work under these conditions is to provide aids that allow them to dynamically execute assigned tasks without depending on continual instruction from Earth. However, achieving this model requires a complex process. Removing the assistance that ground teams provide will likely result in an overwhelmed crew with a higher workload - ground teams have a vast amount of information and expertise, essential to working and living in space, which cannot be neatly codified.

This inevitable shift from the current spaceflight operation model points to the need to support and enable more autonomy among crewmembers. In order to understand future crew needs and contribute to the goal of increasing crew autonomy, we have started to assess crew selfscheduling. In this paradigm shift, a flight controller (specifically the position known as OPS PLAN, or Operations Planner) still provides a schedule for crew, but unlike current International Space Station (ISS) operations, astronauts will be able to reschedule some OPS PLAN assigned tasks and add new ones. Unfortunately, there is limited operational data regarding how crew and ground control would adapt to this shift. This paper will specifically address how we have begun investigating the acceptability of crew self-scheduling for spaceflight operations.

\section{Crew Autonomy}

Autonomy, simply stated, is the ability to make independent decisions on whether, when, and how to do a task $[1,2]$. Thus, the concept of crew autonomy often centers on supporting astronauts with completing tasks at a time other than scheduled, with limited to no assistance of ground support teams. The operational concept of crew autonomy during spaceflight has been discussed and argued since Skylab:

- "...in the future, the ground should give the astronauts the bare framework of a schedule... and 
then let the guys on board figure out the best way of doing them" [3, pp.120]

- "What if a guy starts riding the bicycle ergometer, jiggling the space station, while another guy is taking a long film of a solar flare? ... With so many constraints, I'd say they're bound to screw something up!" [3, pp.122]

Astronauts benefit from having a large team of specialists supporting them with a wide range of expertise as well as monitoring the state of the spacecraft and task progress. Today, teams on Earth and in space work tightly together to plan, schedule, and successfully execute spaceflight operations. While there are some research investigations that have attempted to quantify the effect of crew autonomy [4-6], practical operational application of crew autonomy for spaceflight remains largely unexplored.

Our crew autonomy research is specifically focused on providing astronauts with the ability to determine when they can do tasks in concert with ground support, which we have named self-scheduling. This type of crew autonomy involves exploring autonomy constructs that allows astronauts to weigh in on their schedule, as well as autonomy from mission control teams. Crew self-scheduling is a new operations concept for human spaceflight, and it is significantly different from current ISS operations. On ISS, astronauts have a very detailed schedule to guide them on how and when to complete the various assigned tasks, requiring flight controllers from around the world to verify that all of these tasks can be supported from Earth and in space. To evaluate this new model of crew self-scheduling, we are leveraging Earth analog missions. Earth analogs are field test missions that have some physical similarity to the extreme environment of space, for example hostile or confined environments. Analog missions also provide the opportunity to simulate deep-space exploration conditions, like communication transmission delays and intermittent data links, while still furnishing analogous human spaceflight operational environments. While there is some anecdotal evidence that analog crews can manage daily schedule changes [7], there is limited research on crew's acceptability of self-scheduling and the impact it may have on mission effectiveness and efficiency.

\section{EnABLing Self-Scheduling}

\section{Playbook}

In order to study crew self-scheduling during long duration space missions, astronauts need a tool that allows them to manage their schedules without depending on interaction with mission control. Playbook is a user-friendly, mobileready, comprehensive software tool that enables astronaut crews to manipulate and execute mission plans $[8,9]$. At its core, Playbook is comprised of a mission Timeline that displays the scheduled activities for all crewmembers on any given mission day (Figure 1). Each activity can be selected to reveal the "Stream View," which contains additional details, including associated procedures, execution notes for the crew and operations notes for mission control.

Playbook is unique when compared to other schedule viewers currently in use onboard ISS because it allows astronauts to edit the plan directly in the Timeline without the need for intervention from ground personnel. Over the course of several years, our team has developed and deployed a variety of features that support this unique feature. A pre-selected set of activities can be rescheduled, which are called "flexible" activities. When self-scheduling is enabled, crewmembers can simply drag and drop flexible activities to another time or day (i.e., reschedule); and, flexible activities can be reassigned to other crewmembers by dragging activities across rows in the Timeline. The Scratchpad feature allows activities to be moved across days and views. Crew can also create new activities and schedule them as appropriate within the Timeline. Crew may also add Task List activities, non-critical activities that astronauts are free to schedule into their Timelines if and when they have availability to do so (Figure 2).

When crewmembers update their schedules, each user sees the update in Playbook. Our software tool supports delayed data transmissions between crew and mission control (similar to what we expect to see in exploration-class missions). If an astronaut makes a change, all other crewmembers instantly see the change in the schedule. Mission control also sees the change, but asynchronously, i.e., only after the simulated time-delay has passed. For example, if the Earth analog mission simulates 15-minute communication latency, it would take that length of time for mission control to see an astronaut's change in the schedule.

Playbook can also visualize constraints for astronauts using "no-go zones" in order to address the challenge of indicating activity constraints. One crucial role of mission control is the management of constraints and conflicts in the mission schedule. Limited resources onboard mean that many different, seemingly unrelated activities cannot be scheduled in proximity to each other. For example, a temporal constraint might be that biological samples require time to process; therefore subsequent steps must wait a certain amount of time before they can take place. With the variety of ISS experiments and the limited resources onboard (e.g., crew time, power, video channels), maintaining awareness of all the different types of procedure constraints is a fulltime job typically handled by the OPS PLAN [10]. Since astronauts are not expected to have the operational knowledge held by dozens of individuals in mission control, Playbook provides no-go zones, grayed-out areas on the timeline that indicate where certain activities cannot be scheduled due to an existing constraint (Figure 3). This visualization of activity constraints may decrease cognitive load on the astronauts while they conduct self-scheduling. 


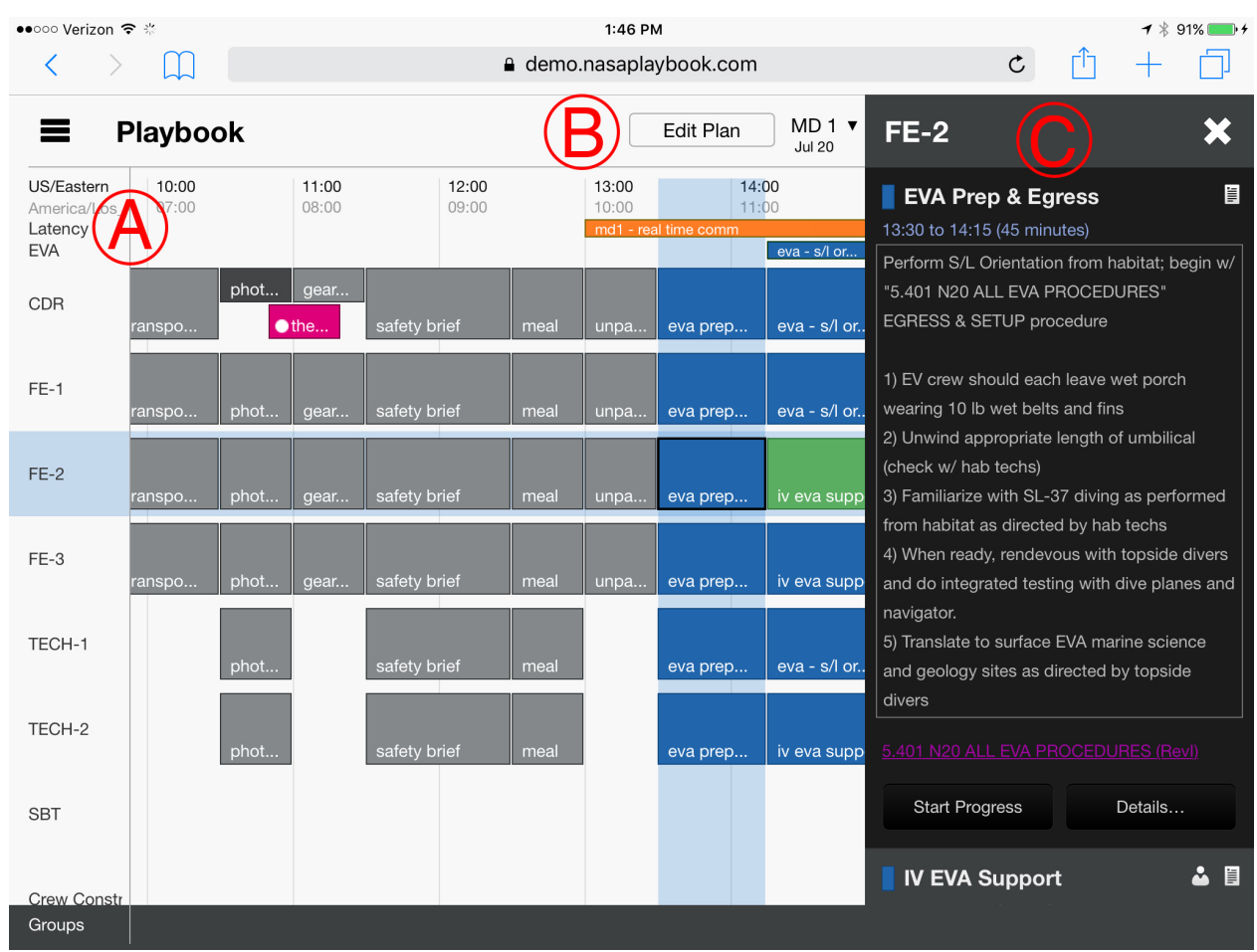

Figure 1. Playbook timeline and activity details: (A) Mission Timeline with crewmembers and activities; (B) Edit Plan feature allows crew to self-schedule; (C) Stream View contains additional activity details

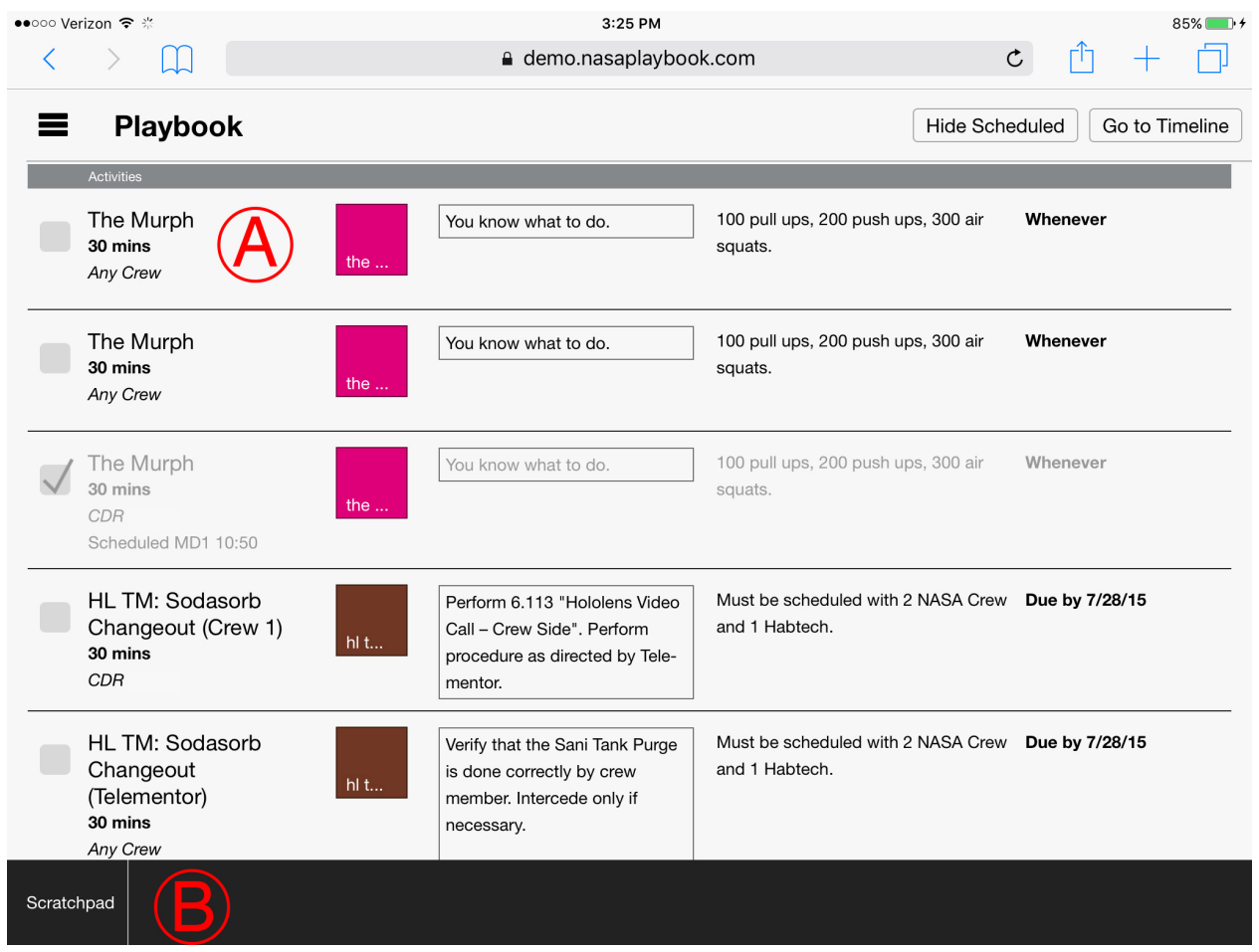

Figure 2. Playbook Task List: (A) Task List contains non-critical activities; (B) Scratchpad allows crew to move activities between views and days 


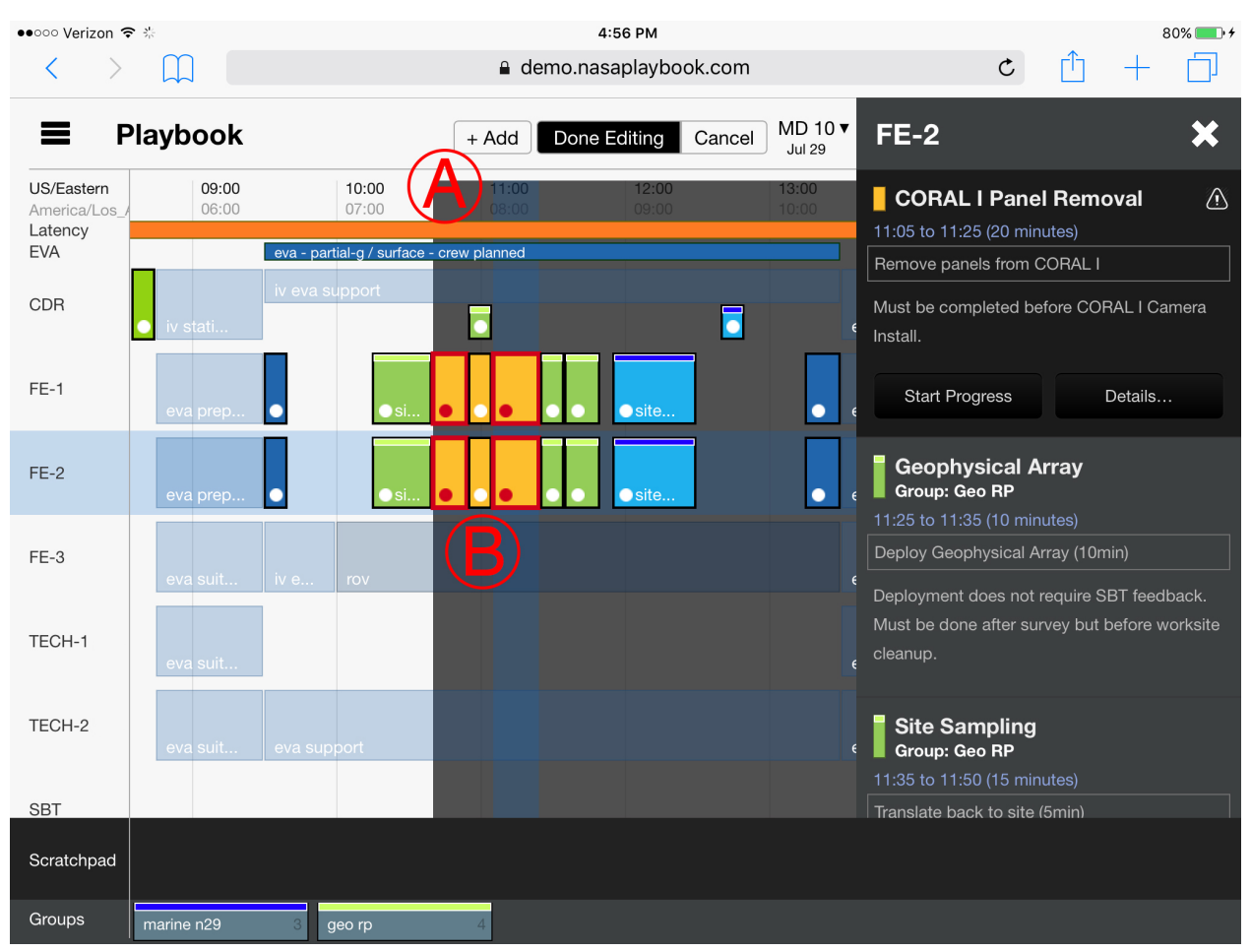

Figure 3. Playbook no-go zones and activities in violation (A) No-go zones in gray depict where activities cannot be scheduled (B) Activities with violations of no-go zones appear with red borders

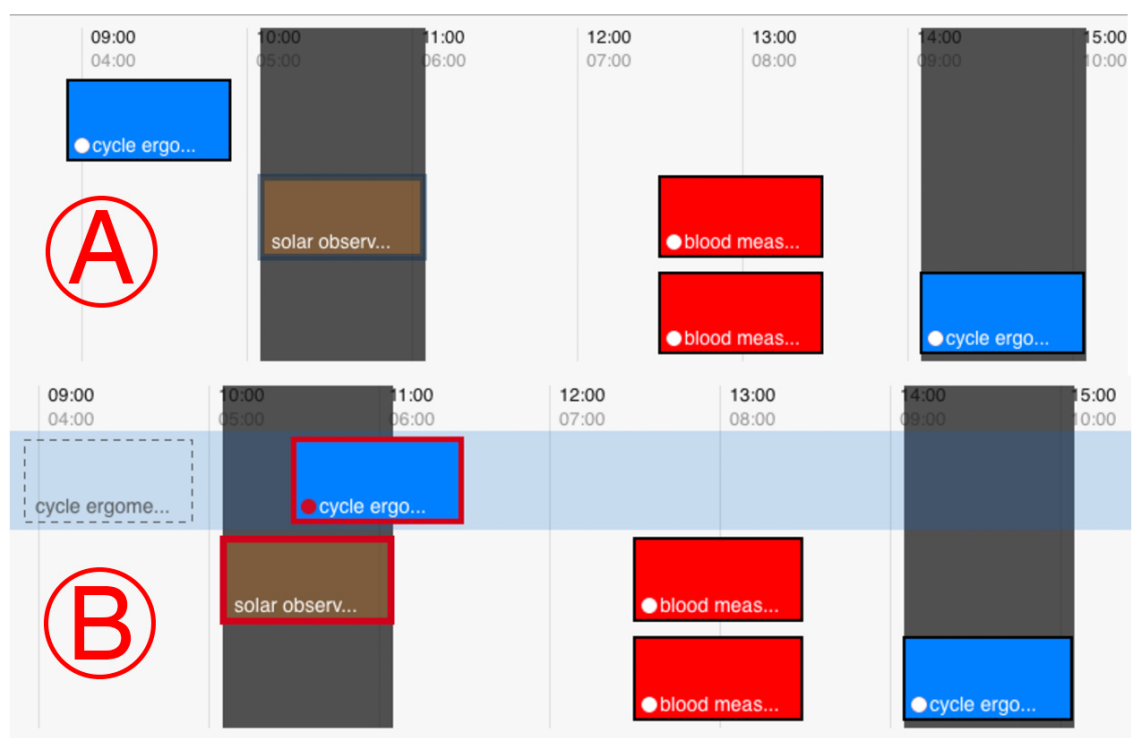

Figure 4. Skylab planning example with Cycle Ergometer's scheduling violations: (A) Cycle Ergometer's no-go zones; (B) Cycle Ergometer activity in violation with Solar Observation activity

Finally, Playbook does not prevent crew from rescheduling activities. However, if the activity is found to not respect the modeled scheduling constraint, it shows a violation. A plan violation (i.e., an activity scheduling that is incorrect and not feasible for execution) is shown with a red outline, red dot, and warnings in the details view. Often, one or more activities are in conflict, and all the dependent activities are shown to have a violation. It becomes the responsibility of the crew to fix the schedule.

\section{Procedural Aids}

In addition to all the Playbook features that enable crewmembers to add, reschedule, and reassign activities, we have tested different procedural aids as well. Specifically, we have generated step-by-step self-scheduling procedures, generated paper guides aimed at aiding strategic planning (e.g., planning over the course of days), and self-schedule matrices. The self-schedule matrices were proposed by OPS 
PLAN as a way of listing and capturing in a paper procedure all the constraints on a specific activity. While many constraints can be visualized in Playbook through the no-go zones, not all the constraints can be currently modeled. Occasionally, crewmembers would have to reference this document in order to confirm planning constraints.

\section{Extending Skylab Example}

In order to illustrate crew self-scheduling, Figure 4 shows how Playbook would have handled the previously cited Skylab example of competing constraints between ergometer versus filming. Playbook shows the crew where the activities (in this case, the Cycle Ergometer activity in blue) can and cannot be placed. For this example, the Cycle Ergometer cannot be scheduled at the same time as Solar Observation, nor if another astronaut is scheduled to use the ergometer. If the activity is placed in the no-go zone, Playbook will indicate if any constraints are being violated, enabling them to reschedule when immediate ground input is not available. In Figure 4, Cycle Ergometer has been rescheduled to occur at the same time as a Solar Observation; hence, both activities are outlined in red since they cannot occur at the same time.

To revise the Skylab example for a Mars-orbiting spacecraft, an opportunistic video of a solar flare or a dust devil on the surface might occur as a crew member is about to start exercising and causing vibration. As the crewmate is starting the opportunistic science, the crew could reschedule both of their activities and have the first crewmember get an early start on some activity that had been scheduled for later in the day if there are no constraints preventing it from starting early. The observation could be over by the time a message could reach Earth and a reply reach Mars.

\section{INITIAL ASSESSMENT: NEEMO}

Over the last three years, we have explored and evaluated crew self-scheduling through Playbook in the NASA Extreme Environment Mission Operations (NEEMO) analog missions. NEEMO is an analog mission where astronauts, scientists, and engineers live and work inside of Aquarius Reef Base, the world's only underwater research station. The NEEMO crew operates in an extreme environment, a confined pressurized vessel 60 feet underwater, and follows NASA's mission operation processes including a Mission Control Center with console positions such as Mission Director, Planner, EVA (Extravehicular Activities), Payload Specialists, etc. The aquanauts perform analogous EVAs through dives on the ocean seafloor, emulating planetary conditions such as micro, Mars, or Moon gravity using weights and flotation devices. Analog operations are performed in coordination with Mission Control and Science Team, with communications at various time-delay conditions, from realtime to 15 minutes one-way light time.

At NEEMO, we have been able to observe and evaluate self-scheduling in an analogous spaceflight operational environment. Key to that process has been Playbook's role as the primary mission-operations and plan-execution tool for Mission Control and the crew. Over four NEEMO missions (NEEMO 18 - 21), we have incrementally included different Playbook features that have enabled the crew to reschedule, plan, and add new activities to their schedule.

NEEMO 18 - Playbook debuted the ability to self-schedule activities in the timeline. The plan contained flexible activities where the constraints were spelled out in plain text in the notes section of the interface. The crew was able to move a few flexible activities in time and reassign them. Flexible activities included interior science experiments as well as Just-In-Time Training opportunities that were assigned to a single crewmember and had simple or no constraints. The crew was observed rescheduling timeinsensitive activities like meals, surveys, and certain experiments.

NEEMO 19 - Playbook included no-go zones to visualize an activity's constraints on the timeline. With this feature, the crew was given the opportunity to move activities with more complex constraints. Mission Control encouraged the crew to move flexible activities to the actual time that they were performed for situational awareness and to see the plan as it is being executed. Flexible activities included Just-InTime Training, habitat monitoring, and interior science experiments. For one of the EVAs, the crew rearranged the tasks and the order of geographical sites visited during the dive, while respecting temporal constraints of the tasks within a site.

NEEMO 20 - The Task List and Scratchpad were introduced to Playbook. The Scratchpad gives the crew the ability to stage activities and easily move them between days, as well as move new activities between the Task List and the Timeline. The Planner added to the Task List a set habitat monitoring activities to be scheduled by the crew. These activities were required, but their scheduling was flexible. The crew also requested more activities to be added to the Task List for them to self-schedule during their free time, such as exercise and personally coordinated events. Groups of activities were introduced as well, allowing crew to move a set of activities at once and preserve the temporal relationship between them.

Using activities in the Task List and activity groups, the crew planned two EVAs during the mission, allowing them to select the geographical sites and rearrange the tasks while still adhering to the constraints. The activities had a required network of temporal constraints (e.g., "build tower before installing video camera" or "at least 20 minutes between pre-sampling and sampling tasks"). Due to the complexity of the EVAs, additional procedural aids were used for the first time. The crew was given time for strategic planning and they were provided constraint matrices that specified mission objectives to be met. After executing the first EVA, the crew had to replan the subsequent EVA based on what 
had been accomplished on the first EVA and additional new scientific priorities.

NEEMO 21 - Playbook introduced the ability for the crew to add new activities directly to the timeline without Mission Control. The crew independently added follow-on activities to existing scheduled activities, reminders and personal activities. Furthermore, Mission Control included flexible science activities (as opposed to predominantly maintenance activities as in previous missions). As the mission progressed, Mission Control granted more flexibility in activities that took place in the same location (the main hallway of the habitat), which allowed the crew to manage the physical space autonomously.

In terms of EVA planning, crewmembers planned two EVAs, each with a different strategy. In the first EVA, crew were asked to add new activities in the timeline, specifying geographical sites, tasks, and requesting reconnaissance assistance from a remotely-operated underwater vehicle. The planning was meant to be free-form, allowing crewmembers to naturally specify the plan resolution they were comfortable with while still communicating to Mission Control the intent of what they wanted to accomplish during the EVA. In the second EVA, crew were asked to schedule an EVA from a set of given sites, tasks from activities, and groups of activities found in the Task List. For both of these, procedural aids were also supplied as reference materials.

\section{LESSONS LEARNED}

\section{Feedback from Surveys \& Debriefs}

Overall, each NEEMO self-scheduling session was successful; all analog crew teams conducted self-scheduling during an analog mission operation. At the end of each analog mission, surveys with open-ended questions were distributed to the analog crewmembers so that they could provide written feedback about Playbook and selfscheduling. When asked what three aspects of Playbook users like the most, several analog crewmembers identified "self-scheduling" or "ease of self-scheduling" as one of their preferred Playbook capabilities. Even when one crewmember initially expressed reluctance to self-schedule, he subsequently listed self-scheduling as one of his favorite features, taking the lead with regards to rescheduling and replanning EVAs quickly and efficiently. Additionally, one crewmember stated, "Eventually we got faster with selfscheduling and felt it did empower us to arrange the schedule as we thought best."

Surveys also provide a method through which analog crew astronauts can request Playbook features. With regards to self-scheduling, there have been many different requests that speak to the crew's desire to increase the amount of self-scheduling they are able to accomplish. For instance, since self-scheduling was first introduced, astronaut crews have requested the ability to add personal activities, which was implemented subsequently. The very idea that an astronaut would like to "add" an activity, a capability that has never existed in human spaceflight operations, speaks to how empowering crew self-scheduling could be for astronauts. Occasionally, analog crewmembers have mentioned wanting to move activities that were not designated as flexible (e.g., "I'd love to have more flexibility in my time management and the few flex items were a good proof of concept.") One analog crewmember commented on wanting to provide a better schedule for daily science activities. While the reasons may be varied (e.g., rearrange schedule in real time or considering noncritical activities like meals operationally flexible), this feedback is insightful with regards to amount of acceptable flexibility in planned schedule.

Some feature requests speak to the crew's frustrations while self-scheduling. For example, there have been multiple requests for some type of "undo" functionality. Analog crewmembers experienced what most expert flight controller planners know: planning and replanning often requires trial-and-error, identifying what-if scenarios, or simply making changes that result in incorrect or invalid plans. Without this capability, analog crews need to manually reverse any undesirable edits. Recently, analog crews requested "copy \& paste" functionality. Together, these feature requests imply that crew would like to make self-scheduling easier, presumably because they considered self-scheduling a desirable task during operations.

During one of the earlier analog missions, one analog astronaut crew found that it was essential to know exactly how the team's schedule had been modified. Shared awareness of plan changes appears to be important, particularly as real-time self-scheduling is significantly different from ISS operations. Each plan change for ISS must be submitted in writing and approved before implemented. With Playbook, if the activity is flexible, the crew is allowed to reschedule. The lack of approval process opens the door to losing situational awareness. The request resulted in a feature that would notify crew and mission support teams of every self-scheduling change. However, once this feature was implemented, we quickly learned from the surveys that detailed logging of each self-scheduling change was overwhelming. Currently, changes in the schedule notifications are summarized. This hopefully balances the need for shared awareness without the addition of workload overhead.

\section{Plan and Replanning Complexity}

The ease with which analog astronauts are able to complete self-scheduling tasks depends on two main factors: how easily the task can be completed with the given tools, and the complexity of the plan. With regards to enabling selfscheduling, Playbook has focused on making it easy for the crew to reschedule and reassign activities while still creating a plan that abides to the known plan constraints. From the various self-scheduling exercises conducted throughout four NEEMO missions, our observations indicate that communicating constraints to crew is challenging. We have 
noticed that for the crew, planning complexity is driven by the number of planning constraints to be satisfied. In order to communicate constraints, we have provided the crew with embedded notes (in the activity's description), documents that include self-scheduling matrices delineating constraints, and visualizations in Playbook. Unfortunately, there are other constraints which are too complex or for which we lack tools to easily quantify for the crew; namely geospatial constraints (e.g., traversability of terrain) and real-time operational constraints (e.g., support-boat schedules, weather).

Through the no-go zone visualizations, we have attempted to simplify some of the planning constraint problem for the operator. While never explicitly mentioned by analog crews, recordings of self-scheduling events indicate that the presence of such visualizations helps users create violationfree plans. When an activity has a corresponding no-go zone visualization, we have observed that the crew immediately know where (and when) to place activities in the timeline. If they do move an activity in the gray zone, users get immediate visual feedback (a red outline) that there is a constraint violated. Rarely have we observed the crew leave a plan with violations. The other documentation alternatives may only have limited usefulness, and their value is being assessed in another Earth analog mission. Thus, compelling visualizations of constraints appears to be a key element to support self-scheduling.

As previously mentioned, planning and replanning complexity varies. Aside from constraints, there are other attributes that create more challenges for the crew completing self-scheduling tasks. In the earlier NEEMO missions, we learned that "small" activities, i.e., activities of short duration, were tedious and difficult to select and move. In NEEMO 20, since the self-scheduling task was considerably more complex than previous missions, we ensured that self-schedule activities were not "small" (i.e., 5 minutes or longer). Interestingly, this crew chose to switch to laptops to perform some of the self-scheduling despite having being encouraged to complete the task on iPads. While self-scheduling was successfully completed, we hypothesized that there is some perceived threshold of plan resolution or plan granularity for which the crew is most comfortable with while performing self-scheduling. NEEMO 21 EVA self-scheduling attempted to explore if crew had a preferred planning granularity by allowing crew to free-form plan the EVAs; however, we did not observe a significant planning granularity difference between Planner scheduled and the crew created EVAs.

\section{Effects on Operational Processes}

From our assessments, crew self-scheduling or rescheduling can be grouped into two categories: unprompted and prompted. Unprompted self-scheduling events include adding new personal activities into the timeline, adding activities from the Task List, and rescheduling flexible activities. This type of self-scheduling appears to have minimal effect on operational process for both crew and mission ground support teams. Self-scheduling of these events demands little of the crew's time and is often done to reflect actual times that the activities were completed. On other occasions, adding a personal activity quickly communicates to ground teams that a particular time is reserved (i.e., not to schedule other activities at that time) or provides crew with reminders of short-duration activities that must be completed. From the mission ground support team's perspective, flexible activities are non-critical activities that can be scheduled at any time. The only additional workload they impose on ground teams is to make sure that the flexible activities correctly model the resource requirements, and this is done before the mission.

Prompted self-scheduling events are where we asked the analog crew to plan, schedule, and reschedule EVA dives. Inherently, these require more complex planning and impact operational processes. From the crew's point of view, it requires at least an hour of crew time to think about the plan and schedule an EVA. We speculate that most of the time is actually spent thinking and discussing the EVAs. The portion of time spent actually self-scheduling includes time adding notes, creating activities, moving around many activities, and correcting any plan violations. Some of the crew reported that complex self-scheduling took a lot of time. Additionally, we did not tell the crew how they should break down the planning and scheduling process; we encouraged them to work as a team to arrive at a schedule. Our observations indicate the once the crew decided on a high-level plan, they delegated the Playbook implementation itself to one or two crewmembers.

As self-scheduling has become more complicated, we have continued to add more features and processes to simplify it for the crew. For instance, in our last evaluation, the crew was able to add entire groups of activities from the Task List (i.e., multiple activities at once) in order to help minimize the amount of time required to complete self-scheduling. We also have given the crew extra time and simple guidelines to complete strategic discussions before the tactical planning and scheduling exercise. We postulate that shifting planning and scheduling responsibilities from ground to the crew requires more than just an easy-to-use timeline tool, because other factors impact the crew's ability to quickly and effectively create timelines, such as plan complexity or processes.

Additionally, from the mission ground control team's point of view, introducing self-scheduling events required adapting an existing operational workflow to receive planning inputs from crew. We quickly learned that Mission Control needs at least two days to review and vet elicited self-scheduling events; this meant that the crew needed to plan EVAs at least two days before they were executed to assess if the schedule created by the crew is valid and if it works within the constraints of the analog mission (e.g., enough support divers). Within a day, Mission Control needed to decide if the proposed plan was executable, and if not, to correct or modify plans before the start of the next 
mission day. This protocol was established and shared with the crew and ground team.

It is important to note that changes to the operational process were only introduced after a previous selfscheduling exercise created strain on the daily workflow. For example, during one of the self-scheduling exercises, the crew rescheduled an EVA the day before execution. This caused unexpected stress on the Mission Control team as part of the morning was spent quickly reevaluating the support dive plan. In another self-scheduling exercise, ground teams changed significant portions of the crew's proposed plan. While this was communicated to the crew, there was some concern that the crew might perceive Mission Control as ignoring their inputs. There is still much to explore with regards to operational processes that would balance the needs of the crew and mission support ground teams.

\section{Future Evaluations}

From the initial evaluations of Playbook and its selfscheduling features conducted primarily in the NEEMO analog missions, a number of extensions and future evaluation topics for crew self-scheduling have been planned. The original goals for crew self-scheduling with Playbook were limited, lightweight plan editing, primarily concerned with replanning unconstrained activities, moving activities with small numbers of flight rules, and violations that can be resolved by moving a single activity. Future evaluations center around three areas: self-scheduling technology demonstrations on the International Space Station, increasing the complexity of the planning problems given to the crew, and new features that extend the type of planning problems that the crew can manipulate using the Playbook tool.

As part of the ISTAR (ISS Testbed for Analog Research) effort, a series of experiments and technology demonstrations conducted on ISS evaluating future exploration concepts, Playbook was identified as a key tool for the use of evaluating crew autonomy on ISS. Playbook was uplinked and deployed in August 2015 and selected to be evaluated during the ESA-SDM (European Space Agency Short Duration Mission), an 8-day ISS mission that occurred in September of 2015. Although the tool was verified on ISS with a ground and crew checkout in August of 2015, it was not used during the ESA-SDM due to the mission being shortened. Playbook is now slated to be used on ISS as part of the CAST (Crew Autonomous Scheduling Test) demonstration. The CAST demonstration will evaluate autonomous planning and replanning on board ISS by the crew and will use Playbook to self-schedule and execute that plan. The expected outcomes of CAST are to inform the design and planning process of future deep space missions. CAST is expected to start at the end of 2016 and will continue into early 2017. These objectives will test and evaluate many of the same Playbook features that the crew used in the NEEMO mission while interacting with
International Space Station plans and in real spaceflight operational conditions.

Since our initial evaluations of self-scheduling, more complex exercises have been planned in other Earth analog missions in order to evaluate the extent of the planning problems that can be given to the crew. The complexity of the exercises evaluate planning problems that have a higher number of activities, more constraints between activities, manipulations involving groups of activities, planning across days, reassigning activities between crewmembers, and having teams collaboratively work on a planning problem versus conducting them individually. Because of the number of activities that need to be planned and their associated constraints we have been looking at different ways of presenting this information. Some current strategies that have been tried include separate self-scheduling matrices, human-readable constraint information inside the activities, and the use of constraint visualizations. This work is ongoing and started as a research task under HERA (Human Exploration Research Analog) Campaign 3.

In addition to more complex exercises, we have explored future enhancements to the Playbook tool that would aid in crew self-scheduling. One of the biggest barriers with the current design of the crew autonomy features is that they are optimized for individual activity manipulation with small amounts of constraints. These features start to become less useful as the number of constraints increase, primarily because the current no-go zone constraint visualization feature shows a hill climbing local search solution to the selected activity's constraints. This means that in a complex planning problem, the user may move an activity into what looks like a solution space, when in fact this will actually cause an additional violation with another activity. A complete solution to mitigate this problem would be to add in a full planning engine that can provide a more thorough search of the solution space, significantly increasing the complexity of the tool. Another way to mitigate this is adding visual features that convey more complex plan constraint information to the user to aid in planning. One method we will explore is to a new visualization to help identify culprits, i.e., the activities associated through constraints violated with the activity in question. Through this and other visual aids, we expect to be able to increase the complexity of plans that can be solved by the crew through Playbook without the complexity of adding in a full planning engine.

\section{CONCLUding ReMARKS}

Crew autonomy will be an essential part of future exploration missions. Through our research, we have assessed how crew self-scheduling may enable crew autonomy. After four NEEMO analog missions, the evidence suggests that self-scheduling is a feasible operational concept. However, much remains to be determined with regards to improving simplicity of selfscheduling, be it at the planning level or at the scheduling interface, and developing operational processes and 
workflows that support crew as well as mission support teams. Our upcoming research will focus on further examining the balance of responsibilities between crew and ground teams as we evaluate self-scheduling plan complexity and self-scheduling in a real spaceflight operational environment.

\section{ACKNOWLEDGEMENTS}

The authors thank all the NEEMO analog crewmembers who participated and provided their unique and diverse insights to crew autonomy through Playbook. NASA Human Research Program through Directed Research Project within Space Human Factors and Habitability funded this research.

\section{REFERENCES}

[1] Caldwell, B.S. and Onken, J.D. (2011) "Modeling and Analyzing Distributed Autonomy for Spaceflight Teams" 41st International Conference on Environmental Systems, 17-21 July 2011, Portland, OR.

[2] Hackman, J. R., \& Oldham, G. R. (1975). "Development of the Job Diagnostic Survey" Journal of Applied Psychology, 60, 159-170.

[3] Cooper, Jr., H.S.F. (1976) A House in Space New York: Holt, Rinehart \& Winston.

[4] Kanas, N., Saylor, S., Harris, M., Neylan, T., Boyd, J., Weiss, D.S., et al. (2010) "High versus low crewmember autonomy in space simulation environments" Acta Astronautica, 67, pp. 731-738.

[5] Kanas, N., Harris, M., Neylan, T., Boyd, J., Weiss, D., Cook, C., and Saylor, S. (2011) "High vs. low crewmember autonomy during a 105-day Mars simulation mission" Acta Astronaut. 69. pp. 240-244.

[6] Roma, P.G, Hursh, S.R., Hienz, R.D., Emurian, H.H., Gasior, E.D., Brinson, Z. S., Brady, J.V. (2011) "Behavioral and biological effects of autonomous versus scheduled mission management in simulated spacedwelling groups" Acta Astronautica, 68 (9-10), pp. 15811588 .

[7] Love, S.G. and Harvey, R.P. (2014) "Crew autonomy for deep space exploration: Lessons from the Antarctic Search for Meteorites" Acta Astronautica, 94, pp. 83-92.

[8] Marquez, J.J., Pyrzak, G., Hashemi, S., McMillin, K., Medwid, J., et al. (2013) "Supporting Real-Time Operations and Execution through Timeline and Scheduling Aids" $43^{\text {rd }}$ International Conference on Environmental Systems, 14-18 July 2013, Vail, CO.

[9] Hashemi, S. and Hillenius, S. (2013) "@NASA: The User Experience of a Space Station." SXSW Interactive 2013. SXSW. SXSW Interactive, Austin, TX. 10 Mar. 2013. Speech.
[10] Marquez, J.J. (2015) "Integrating human performance measures into space operations: Beyond our scheduling capabilities?" IEEE Aerospace Conference, doi:10.1109/AERO.2015.7119112

\section{BIOGRAPHY}

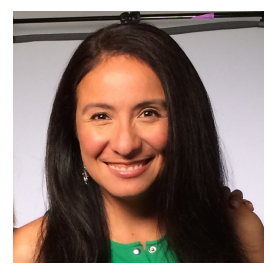

Jessica J. Marquez received a B.S.E. in Mechanical Engineering from Princeton University, followed by a S.M. from the Department of Aeronautics and Astronautics at MIT. She received her Ph.D. in Human Systems Engineering from MIT. Since 2007, she has been working at the NASA Ames Research Center within the Human Systems Integration Division. As part of the Human Computer Interaction Group, she has supported the development and deployment of various instances of SPIFe (Scheduling \& Planning Interface For exploration), the planning and scheduling software tool for several space missions, including the International Space Station. As the Human Research Program's Discipline Science for the Risk of Inadequate Design of Human and Automation/Robotics Integration, she continues to conduct research in the field of human-automation integration, human-computer interaction, and space human factors engineering.

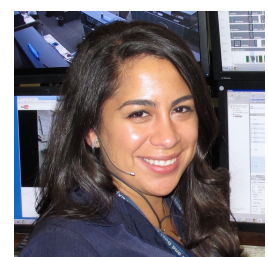

Ivy Deliz is a software developer in the Playbook team. She has a Bachelor's degree in Computer Science from the University of Puerto Rico, Rio Piedras, and a Master's in Computer Science (Vision and Graphics) from Columbia University. She developed SPIFe planning and scheduling tools for various International Space Station Mission Control consoles, Mars Science Laboratory (Curiosity) and LADEE Lunar Orbiter. She has been the Lead Planner for the NEEMO Analog Mission since NEEMO 18 and has trained aquanauts on Playbook use for mission operations. She also supports the BASALT (Biologic Analog Science Associated with Lava Terrains) Analog Mission for on-site technical support.

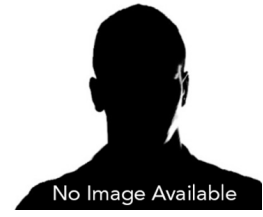

Bob Kanefsky is a software engineer and part of the Playbook development team. Previously he contributed to the SPIFe set of software applications for planning and scheduling human and robotic activities: Phoenix Science Interface for the first successful Mars polar lander, LASS for the LADEE lunar orbiter, MSLICE for the Curiosity rover, and Score and APEX for ISS Mission Control Houston. He provided on-site support for the initial months of three Mars missions: Pathfinder, Spirit, and Phoenix. He holds a bachelor's degree in Formal Systems from Stanford University. 


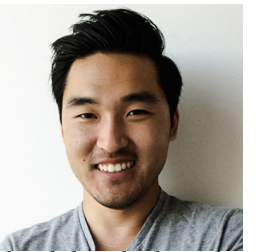

Jimin Zheng is a user experience researcher and designer on the Playbook team. He designed many of the key self-scheduling features in Playbook evaluated during several of the NASA Analog missions. He has dual bachelor degrees from the University of California, Irvine in Cognitive Psychology and Business Administration and also holds a Master of HumanComputer Interaction from Carnegie Mellon University. Jimin is currently a NASA contractor working under San Jose State University Research Foundation.

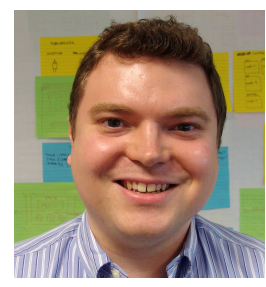

Steven Hillenius leads the Playbook/SPIFe team at NASA Ames Research Center. As part of his work as a Principal Investigator for an HRP research proposal on onboard planning he has led the design and development of Playbook from a plan viewer into a crew autonomy tool. Previously he did product design and conducted user research for Score, MSLICE, and LASS, a series of SPIFe mission planning tools used for International Space Station crew planning, Mars Science Laboratory (Curiosity) rover planning, and LADEE (Lunar Atmospheric Dust Environment Explorer) orbiter planning. Steve holds a Masters in HumanComputer Interaction and a B.S. in Information Systems with an additional major in Human-Computer Interaction, both from Carnegie Mellon University.

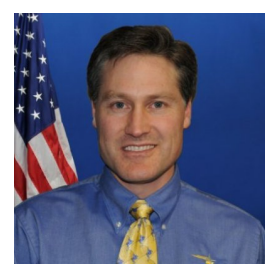

Marcum Reagan serves as CAPCOM for the International Space Station (ISS) Mission Control, communicating with astronauts in orbit. He was an aquanaut on the NASA Extreme Environment Mission Operations 2 (NEEMO 2) crew, and subsequently served as Mission Director for NEEMO missions. As Station Training Lead in Mission Operations, he lead of instructors who are responsible for developing and executing complex simulations for assembly and operations. 